\title{
How has the COVID-19 pandemic affected tobacco users in India: Lessons from an ongoing tobacco cessation program
}

\author{
Himanshu A. Gupte', Gauri Mandal², Dinesh Jagiasi
}

\begin{abstract}
INTRODUCTION Tobacco use is detrimental at any time. However, it is proving to be more dangerous during the COVID-19 pandemic. Tobacco use may increase the risk of being infected, increases the chances of complications, and also increases the probability of its spread. We assessed the awareness about this association and the impact of the lockdown on tobacco use among tobacco users registered before the lockdown for LifeFirst, a tobacco dependence treatment program.

METHODS 1016 tobacco users were under active follow-up in their course of the 6-month counselling program. From 14 to 28 May 2020, 650 (64\%) of these registered users were contacted by counsellors for follow-up sessions over the telephone. Semi-structured questionnaires were filled in during the calls.

RESULTS Two-thirds (67\%) of tobacco users were unaware of the association between tobacco and COVID-19. Only 30\% of the users felt that the current situation had affected their tobacco use, the commonest impacts being unavailability and increased prices of tobacco products. While this was seen as an opportunity to quit by some users, some reported increased tobacco use due to increased stress. Of the 219 (34\%) tobacco users who quit tobacco during the lockdown, 51\% quit because of the lockdown and their concern over COVID-19. Abstinence among those who were aware of the association between the coronavirus and tobacco was twice that among those who were not aware.

CONCLUSIONS Awareness activities about the harmful effects of tobacco during the coronavirus pandemic have to be strengthened. Measures to motivate and support tobacco users to quit have to be provided through cessation services.
\end{abstract}

\section{AFFILIATION}

1 Narotam Sekhsaria Foundation, Mumbai, India

2 Salaam Bombay Foundation, Mumbai, India

\section{CORRESPONDENCE TO}

Gauri Mandal. Salaam Bombay Foundation, Nirmal Building, Nariman Point, Mumbai, 400025 India. E-mail: gauri.mandal34@ gmail.com

\section{KEYWORDS}

counseling, tobacco cessation, COVID-19, lockdown

Received: 25 June 2020 Revised: 7 August 2020 Accepted: 1 September 2020

\section{INTRODUCTION}

Tobacco use continues to be one of the biggest public health threats ${ }^{1}$. Tobacco damages the immune system and impairs lung function, making users more prone to respiratory infections ${ }^{1,2}$. COVID-19 is an infectious disease that mainly attacks the lungs. During this pandemic, tobacco is proving to be more harmful than ever.

There is growing evidence that smoking is associated with adverse outcomes and increased severity of COVID-19. Smokers have a higher risk of contracting coronavirus infection compared to non-smokers ${ }^{3-5}$. Smoking may also raise the risk of COVID-19 by increasing enzymes that allow coronavirus to enter and damage lung cells ${ }^{6,7}$.

A high proportion of smokers have health conditions such as Chronic Obstructive Pulmonary Disease (COPD) and heart disease. For them, catching the virus may increase their risk of developing complications and may prove to be potentially fatal. Indeed, coronavirus is known to have severe complications and even higher mortality in people with comorbidities like hypertension, diabetes, and COPD, which are also associated with smoking ${ }^{8,9}$.

Use of any tobacco product also increases the 
risk of being infected by coronavirus and increases the probability of its spread. This may be due to contaminated fingers or tobacco products touching the face and mouth for smoking as well as consuming smokeless tobacco. Sharing tobacco products like cigarettes, bidis, e-cigarettes, hookah etc., and social customs of preparing for others khaini or mawa mixture (smokeless tobacco products), increases contact and the risk of transmitting the virus ${ }^{10}$.

Smokeless tobacco users may facilitate the spread of the disease through spitting. COVID-19 can spread through small droplets from the nose or mouth, which are dispersed when an infected person coughs, sneezes, talks, exhales or spits ${ }^{10,11}$.

Considering this, a number of Indian states have partially or completely banned tobacco use and spitting in public places. Some states and districts have also temporarily banned the production or sale of tobacco products ${ }^{12}$. The Government of India declared a lockdown in the country since 24 March and extended it to 30 May 2020. It was lifted from 1 June in phases, depending on the severity of the situation in individual cities, districts or states.

LifeFirst is a tobacco dependence treatment programme initiated by Narotam Sekhsaria Foundation in collaboration with Salaam Bombay Foundation that offers tobacco cessation counselling by experienced counsellors. Based on available evidence on the association of tobacco use and COVID-19, and to understand its probable impact on tobacco use behaviour, we assessed awareness about the association between tobacco and COVID-19 infection and the impact of the lockdown on tobacco use among tobacco users registered for LifeFirst services before the lockdown.

\section{METHODS}

Current tobacco users are identified and motivated to make quit attempts by registering for a 6-month counselling program. Written informed consent is obtained from them before registering for the program. An initial, detailed face-to-face counselling session is followed by six follow-up sessions over six months through a combination of in-person and telephone counselling.

At the beginning of the lockdown, a total of 1016 tobacco users were under active follow-up of LifeFirst counsellors across all the settings in four states of India. The registered individuals included factory workers from the workplace cessation program, and patients were referred from outpatient as well as inpatient departments of government and charitable hospitals. Majority of the registered individuals, enrolled into the free counselling service, belong to the lower socioeconomic strata. Users were in various stages of follow-up in their course of the 6-month tobacco dependence treatment counselling.

During the period 14 to 28 May 2020, 650 (64\%) of the registered users were actively contacted by the respective LifeFirst counsellors for routine follow-up sessions over the telephone. The LifeFirst team comprised seven trained counsellors. During these follow-up counselling calls, the clients were asked specifically about their awareness regarding the relationship between their tobacco use and coronavirus infection, whether the pandemic and the lockdown had impacted their tobacco use and in what way, and if they had stopped using tobacco in this particular period, and was it due to the current situation.

Verbal consent to document this additional information was taken during the follow-up counselling call. Semi-structured questionnaires (Supplementary file) were filled in during the calls by the counsellor and later converted into electronic version by entering into Microsoft Excel. Proportions were used for analysis and no statistical tests were applied.

\section{RESULTS}

Among the 650 tobacco users who were interviewed, $46 \%$ were smokers, $45 \%$ smokeless tobacco users, and $9 \%$ were dual users. The majority (96\%) were males and their average age was 40 years. It was found that $67 \%$ were not aware of the association between tobacco and COVID-19. Among those who were aware, the knowledge regarded: 1) the lowered immunity due to tobacco use making the user prone to infection, 2) sharing of tobacco products and resultant increased risk of infection, and 3 ) that smoking affects lung health and makes the smoker prone to coronavirus infection.

Almost $70 \%$ of the users felt that the current situation had not affected their tobacco use. Among those who felt that it had impacted their tobacco use, 
the commonest impacts were: 1) non-availability of tobacco products $(45 \%), 2)$ increased price of tobacco products $(27 \%), 3)$ some users felt that this was an opportunity for them to quit (24\%), and 4) a small proportion $(4 \%)$ of users reported increase in their tobacco use due to the increased stress that they were experiencing in the current context.

A total of 219 tobacco users (38\% of those contacted) reported to have abstained from using tobacco after the lockdown started. The proportion of users who abstained among those who were aware about the association of COVID-19 and tobacco use was twice $(51 \%)$ that among those who were not aware $(25 \%)$.

The main reasons for stopping tobacco use were related to the lockdown or concerns about the coronavirus $(51 \%)$. The proportion of smokers, smokeless tobacco users and dual users who stopped their use due to the lockdown was $13 \%, 22 \%$ and $18 \%$, respectively, with the overall proportion being $17 \%$ (Table 1). The main motivation for the rest of the users who had stopped their tobacco use was their health condition (not relating it to coronavirus) or as an impact of the LifeFirst counselling programme itself.

Table 1. Assessment of knowledge and impact of coronavirus and the lockdown on tobacco use among tobacco users* from four states of India, May 2020 $(\mathrm{N}=1016)$

\begin{tabular}{lrr} 
& $n$ & $\%$ \\
\hline Total ongoing clients & 1016 & 100 \\
Total interviewed & 650 & 64 \\
Not aware about association between tobacco & 436 & 67 \\
and coronavirus & & \\
Coronavirus/lockdown had an impact on & 197 & 30 \\
tobacco use & & \\
Impact on tobacco users & 54 & 27 \\
Increased price & 88 & 45 \\
Tobacco not available & 47 & 24 \\
Opportunity to quit & 8 & 4 \\
Increased use due to increased stress & 219 & 34 \\
Stopped tobacco use during lockdown & 110 & 51 \\
Among those aware about the association & 109 & 25 \\
Among those not aware about the association & 112 & 51
\end{tabular}

* Registered for counselling in LifeFirst program before the lockdown.

\section{DISCUSSION}

Our findings suggest that the lockdown enforced by the government can be considered as an opportunity to quit tobacco. The study showed that people are making quit attempts during this period. The World Health Organisation recommends promotion of proven cessation interventions to support tobacco users during this period ${ }^{5}$. Thus, it is important to offer cessation services that are accessible to tobacco users during the pandemic.

The findings also highlight the fact that the majority of the tobacco users who enrolled for the cessation program were not aware about the association between tobacco use and COVID-19. However, the data also suggest that the probability of stopping tobacco use was doubled by the self-reported presence of this awareness. This demonstrates the need for creating awareness about the harmful effects of tobacco use during this pandemic. While it is known that about half of all tobacco users in India are willing to quit, a small proportion of these make quit attempts and very few are actually successful ${ }^{13}$. Increasing awareness about the influence of tobacco use on the risks of COVID-19 coupled with improved access to cessation services, like quitlines through toll-free numbers, mobile based cessation programmes etc., will improve motivation to quit and translate into higher number of assisted quit attempts.

Increased prices and non-availability of tobacco products for extended periods are forcing users to take a long-term decision to quit tobacco. This positive change of behaviour supports the theory that raising tobacco taxes and banning certain tobacco products help to reduce the prevalence of tobacco use ${ }^{14}$.

An important aspect to note here is the availability of certain products despite a ban. This points to lapses in implementation and regulation that need to be tackled by authorities or mechanisms beyond the health system.

Effectiveness of phone-based counselling cessation services is also demonstrated by the sustained quit attempts among those who were neither aware about the association of coronavirus and tobacco use nor did they stop their use due to the unavailability or increased prices.

Many people are and will be facing issues like 
fear, anxiety etc. due to the current environment and to tackle these, people may recourse to tobacco, alcohol or other psychoactive substances. This can be studied further by undertaking in-depth studies to assess the changes in substance-use behaviour. Some tobacco users may initiate these habits during this period while some may relapse or increase consumption. Therefore, mental health issues resulting from the current situation should be studied and addressed. Furthermore, easily accessible comprehensive interventions including awareness and cessation support counselling are needed.

\section{Strengths and limitations}

Our study has several strengths. To the best of our knowledge, this is the first operational research study conducted in India to assess the impact of the pandemic and the lockdown on the behaviour of tobacco users. Another highlight is that the sample included smokeless tobacco users as well. The respondents were from various parts of the country and from the lower socioeconomic strata, thus representing the majority of tobacco users in India. There are a few limitations of the study. Since the study was conducted among tobacco users already enrolled in a cessation program, the sample is not representative of all tobacco users. Another limitation is that tobacco use status was self-reported and not validated biochemically.

\section{CONCLUSIONS}

The extent of awareness about the association of tobacco and COVID-19 influenced the tobacco users to stop tobacco use. Awareness activities about the harmful effects of tobacco during the pandemic have to be prioritised and further strengthened. Creating an environment with reduced access to tobacco products will augment reduction of tobacco consumption to some extent. The lockdown or the pandemic can prove to be an ideal time to quit tobacco use and people are making quit attempts. Adequate measures to motivate and support tobacco users to quit have to be provided through promotion of existing quitlines and improved access to other cessation services.

\section{REFERENCES}

1. U.S. Department of Health and Human Services. The
Health Consequences of Smoking: 50 Years of Progress. A Report of the Surgeon General. Atlanta, GA: U.S. Department of Health and Human Services, Centers for Disease Control and Prevention, National Center for Chronic Disease Prevention and Health Promotion, Office on Smoking and Health; 2014. https://www.ncbi.nlm.nih. gov/books/NBK179276/pdf/Bookshelf_NBK179276. pdf. Accessed August 7, 2020.

2. Zhou Z, Chen P, Peng H. Are healthy smokers really healthy? Tob Induc Dis. 2016;14(November):1-12. doi:10.1186/s12971-016-0101-z

3. Vardavas CI, Nikitara K. COVID-19 and smoking: A systematic review of the evidence. Tob Induc Dis. 2020;18(March):1-4. doi:10.18332/tid/119324

4. Patanavanich R, Glantz SA. Smoking Is Associated With COVID-19 Progression: A Meta-analysis. Nicotine Tob Res. 2020;22(9):1653-1656. doi:10.1093/ntr/ntaa082

5. World Health Organization. WHO statement: Tobacco use and COVID-19. https://www.who.int/news-room/ detail/11-05-2020-who-statement-tobacco-use-andcovid-19. Published May 11, 2020. Accessed June 13, 2020.

6. Berlin I, Thomas D, Le Faou AL, Cornuz J. COVID-19 and smoking. Nicotine Tob Res. 2020;22(9):1650-1652. doi:10.1093/ntr/ntaa059

7. Gretler C. Smoking Helps Open Gateway to Coronavirus Infection. https://www.bloombergquint.com/ coronavirus-outbreak/smoking-helps-open-gateway-tocoronavirus-infection-study-shows. Published April 9, 2020. Updated April 10, 2020. Accessed June 13, 2020.

8. Zhao Q, Meng M, Kumar R, et al. The impact of COPD and smoking history on the severity of Covid-19: A systemic review and meta-analysis. J Med Virol. 2020. doi: $10.1002 / j m v .25889$

9. Guan WJ, Liang WH, Zhao Y, et al. Comorbidity and its impact on 1590 patients with Covid-19 in China: A Nationwide Analysis. Eur Respir J. 2020;55(5):2000547. doi:10.1183/13993003.00547-2020

10. World Health Organization. Q\&A: Tobacco and COVID-19. https://www.who.int/emergencies/diseases/ novel-coronavirus-2019/question-and-answers-hub/q-adetail/q-a-on-smoking-and-covid-19. Published May 27, 2020. Accessed June 13, 2020.

11. Harvard T. H. Chan School of Public Health. Tips for Smokers and Tobacco Users. https://www.hsph.harvard. edu/india-center/tips-for-smokers-and-tobacco-users/. Accessed June 13, 2020.

12. Singh A, Chaturvedi P. Smokeless tobacco use and the COVID-19 pandemic. Blog | BMJ Journals. https://blogs. bmj.com/covid-19/2020/04/08/smokeless-tobacco-useand-the-covid-19-pandemic/. Published April 8, 2020. Accessed June 13, 2020.

13. Tata Institute of Social Sciences (TISS), Mumbai and Ministry of Health and Family Welfare, Government of India. Global Adult Tobacco Survey GATS 2 India 
2016-17. New Delhi, India: Ministry of Health and Family Welfare, Government of India, World Health Organization, Tata Institute of Social Sciences; 2018. http://download.tiss.edu/Global_Adult_Tobacco_ Survey2_India_2016-17_June2018.pdf. Accessed August 7, 2020.

14. World Health Organization. Tobacco Free Initiative (TFI): Other economic issues in tobacco control. https://www. who.int/tobacco/economics/other_issues/en/. Accessed July 30, 2020.

\section{ACKNOWLEDGEMENTS}

We gratefully acknowledge the cooperation of the study participants and the efforts of the LifeFirst intervention team: Sadashiv Hattarki, Asha Kamble Shridhar Mulam, Nitesh Agre, Mayuresh Jagtap, Neha Korlekar, Harshali Gaikwad and Grishma Shah.

\section{CONFLICTS OF INTEREST}

The authors have completed and submitted the ICMJE Form for Disclosure of Potential Conflicts of Interest and none was reported.

\section{FUNDING}

There was no source of funding for this research.

PROVENANCE AND PEER REVIEW

Not commissioned; externally peer reviewed. 